1126

\section{CARDIAC SCREENING CLINICS- A FEASIBLE TOOL FOR EFFICIENT PATIENT CARE}

R. Chakupurakal, D. Sobithadevi, M. Ahmed,

\section{A. Manzoor}

Dept. of Paediatrics, Queen's Hospital Burton, Burton on Trent, UK

Introduction: Paediatric cardiologist run outreach clinics have long waiting times. An audit identified that significant number of patients referred to our outreach clinic, in district general hospital, had innocent murmurs or symptoms without any cardiac pathology. As a result we set up a cardiac screening clinic, run by an appropriately trained consultant paediatrician, to relieve pressures on the outreach clinic.

Aim: The aim was to analyze the efficacy and safety of the cardiac screening clinic in filtering non- cardiology patients and making appropriate referrals to paediatric Cardiologists.

Methods: Data on all patients referred to the clinic, from in hospital or primary care, was collected and analyzed retrospectively.

Result: 179 patients were seen between May 2007 and March 2010. Of these patients majority were reviewed for murmurs [81\% (145/179)]. Other referrals included syncope, chest pain, palpitations or a positive family history of cardiac disorders [19\% (34/179)]. 70\% (125/179) of all patients were discharged after their initial assessment whilst $4 \%$ $(54 / 179)$ were followed up. Murmur was present in only $56 \%(84 / 145)$ of the patients with murmur, at the time of review. Of these $37 \%$ (31/84) had abnormal echocardiograms, mainly ventricular septum defect [61\% (19/31)] and pulmonary stenosis [35\% (11/31)]. $16 \%$ of all screened patients were referred on to the paediatric cardiologist.

Conclusion: Screening clinics are effective filters to select out patients with no cardiac anomaly. We have been successful in relieving the pressure on the cardiology outreach clinic and hence reduce waiting times.
STANDARDISATION OF REGIONAL NEONATAL PRESCRIBING: EAST OF ENGLAND (EOE) PERINATAL NETWORK

\author{
P. Muthukumar ${ }^{1}$, S. Stone ${ }^{2}$, N. Gooding ${ }^{3}$,
}

K. Farrer ${ }^{2}$, East of England Perinatal Network

${ }^{1}$ Neonatal Unit, Cambridge University Hospital NHS Foundation Trust, ${ }^{2}$ Acute Neonatal Transport Service, East of England, ${ }^{3}$ Paediatric/Neonatal

Pharmacist, Cambridge University Hospital NHS

Foundation Trust, Cambridge, UK

Background: EoE perinatal network has 18 hospitals with 3 lead units. In line with network guidelines the neonatal transport service transfers babies between network lead units depending on the level of care required. This process is prolonged with potential for medication errors during each change as network units use different drug infusion policies and concentrations.

Aim:

1. Survey of our current infusion concentrations used within the network

2. Implementation of unified drug prescription across network.

Method: Between June and October 2009 an email questionnaire followed by telephone survey was sent to pharmacists and local NICU lead nurses in each unit requesting information on the concentrations of their top 10 drug infusions, and types of pumps and syringes used.

Survey results: Data collected from the survey shows wide range of infusion concentrations used for commonly prescribed infusions. The results were discussed in the network board meeting, a proposal for unified prescription was put forward to the clinicians' development group and network user group which reviewed and accepted proposal.. The final proposed unified drug infusion concentrations, with introduction of standard syringe labels will be implemented across the region from April 2010.

Conclusion: With the development of networks and transfer of babies within the network it is important to have a common agreed prescription for infusions, pumps and syringes. We believe this will reduce time for transfers, reduce medication errors and improve the quality of care as well as supporting the national QIPP agenda. 